\title{
Subdominant $\mathrm{H} 60$ antigen-specific CD8 T-cell response precedes dominant $\mathrm{H} 4$ antigen-specific response during the initial phase of allogenic skin graft rejection
}

\author{
Kang Il Yoo ${ }^{1}$, Ji Yeong Jeon ${ }^{1}$, Su Jeong Ryu ${ }^{1}$, Giri Nam ${ }^{1}$, Hyewon Youn ${ }^{2,3}$ and Eun Young Choi ${ }^{1}$
}

In allogeneic transplantation, including the B6 anti-BALB.B settings, $\mathrm{H} 60$ and $\mathrm{H} 4$ are two representative dominant minor histocompatibility antigens that induce strong CD8 T-cell responses. With different distribution patterns, $\mathrm{H} 60$ expression is restricted to hematopoietic cells, whereas $\mathrm{H} 4$ is ubiquitously expressed. H60-specific CD8 T-cell response has been known to be dominant in most cases of B6 anti-BALB.B allo-responses, except in the case of skin transplantation. To understand the mechanism underlying the subdominance of $\mathrm{H} 60$ during allogeneic skin transplantation, we investigated the dynamics of the H60-specific CD8 T cells in B6 mice transplanted with allogeneic BALB.B tail skin. Unexpectedly, longitudinal bioluminescence imaging and flow cytometric analyses revealed that H60-specific CD8 T cells were not always subdominant to H4-specific cells but instead showed a brief dominance before the $\mathrm{H} 4$ response became predominant. H60-specific CD8 T cells could expand in the draining lymph node and migrate to the BALB.B allografts, indicating their active participation in the anti-BALB.B alloresponse. Enhancing the frequencies of H60-reactive CD8 T cells prior to skin transplantation reversed the immune hierarchy between $\mathrm{H} 60$ and $\mathrm{H} 4$. Additionally, $\mathrm{H} 60$ became predominant when antigen presentation was limited to the direct pathway. However, when antigen presentation was restricted to the indirect pathway, the expansion of H60-specific CD8 T cells was limited, whereas H4-specific CD8 T cells expanded significantly, suggesting that the temporary immunodominance and eventual subdominance of $\mathrm{H} 60$ could be due to their reliance on the direct antigen presentation pathway. These results enhance our understanding of the immunodominance phenomenon following allogeneic tissue transplantation.

Experimental \& Molecular Medicine (2015) 47, e140; doi:10.1038/emm.2014.107; published online 13 February 2015

\section{INTRODUCTION}

Minor histocompatibility $(\mathrm{H})$ antigens are peptide fragments derived from proteins with polymorphisms that arise from sequence variations or null/expression of proteins derived from the same genetic locus. ${ }^{1,2}$ Because the polymorphic regions fall into the epitope sequences presented by major $\mathrm{H}$ complexes (MHC), minor $\mathrm{H}$ antigens may be recognized as foreign epitopes during allogeneic cell and tissue transplantation, particularly between MHC-matched individuals, thereby inducing specific CD4 and CD8 T-cell responses. ${ }^{3}$ These alloreactive $\mathrm{CD} 4$ or $\mathrm{CD} 8 \mathrm{~T}$ cells contribute to the rejection of the transplanted allogeneic cells and tissues and to the generation of graft-versus-host disease. ${ }^{4}$ Therefore, understanding the characteristics of CD8 T-cell responses for minor $\mathrm{H}$ antigens would provide valuable insights into controlling tissue rejection and graft-versus-host disease.

When the immune system of an individual encounters multiple epitopes derived from polymorphic alleles of background-disparate individuals, allo-responses are simplified by the immunodominance phenomenon, in which T-cell responses are focused on several peptide/MHC epitopes, though potentially hundreds and thousands of antigenic peptides could be recognized. ${ }^{5}$ Therefore, the responses for some dominant antigens dominate over the responses for others, generating an immune hierarchy among the different epitope specificities of CD8 T-cell responses. ${ }^{6}$ In the alloresponses induced in $\mathrm{C} 57 \mathrm{BL} / 6$ (B6) by the transplantation of cells or tissues originating from BALB.B mice (MHC-matched

\footnotetext{
${ }^{1}$ Department of Biomedical Sciences, Seoul National University College of Medicine, Seoul, Korea; ${ }^{2}$ Department of Nuclear Medicine, Cancer Imaging Center, Seoul National University Hospital, Seoul, Korea and ${ }^{3}$ Tumor Microenvironment Global Core Research Center, Cancer Research Institute, Seoul National University College of Medicine, Seoul, Korea Correspondence: Professor EY Choi, Department of Biomedical Sciences, Seoul National University of College of Medicine, 103 Daehang-ro, Chongno-gu, Seoul, 110-799, Korea.

E-mail: eycii@snu.ac.kr
}

Received 9 October 2014; accepted 22 October 2014 
but multiple-minor $\mathrm{H}$ antigen-mismatched with $\mathrm{B} 6$ mice), a few dominant minor $\mathrm{H}$ antigens have been identified, including $\mathrm{H} 60, \mathrm{H} 4, \mathrm{H} 28, \mathrm{H} 7, \mathrm{H} 13$ and HY, ${ }^{4,6,7}$

In several $\mathrm{B} 6$ anti-BALB.B settings, $\mathrm{H} 60$ and $\mathrm{H} 4$ minor $\mathrm{H}$ antigens have been considered to be two major antigens that induce dominant responses, whereas $\mathrm{H} 13$ and HY-Uty-reactive CD8 T-cell responses are subdominant. ${ }^{6,8,9}$ The CD8 T-cell response against $\mathrm{H} 60$, which is expressed by hematopoietic lineage cells, ${ }^{10}$ is exceptionally dominant in B6 mice immunized with BALB.B splenocytes and during graft-versus-host disease induced in BALB.B mice via the transplantation of B6 bone marrow and spleen cells. ${ }^{8}$ The dominance of H60-specific response was ascribed to the presence of a high precursor frequency of the reactive CD8 T cells in the naïve pool, because of insufficient negative selection against H60-reacitive CD8 $\mathrm{T}$ cells in the thymus of B6 mice. ${ }^{11}$ However, in allogeneic skin transplantation, CD8 T-cell response against $\mathrm{H} 4$ was dominant. ${ }^{9}$ The immunodominance of $\mathrm{H} 4$ was ascribed to the wide distribution of $\mathrm{H} 4$, not only in the hematopoietic cells but also in epithelial cells and other cell types. ${ }^{12}$ Therefore, H4 was considered to be dominant when solid tissue was transplanted, whereas $\mathrm{H} 60$ was dominant when the exposure of allogeneic hematopoietic cells occurred during transplantation. In line with this finding, it was reported that $\mathrm{H} 60$ was dominant during heart transplantation involving primary vascularization, whereas $\mathrm{H} 4$ was dominant in skin transplantation. ${ }^{13}$ However, other than the different antigen distribution between $\mathrm{H} 60$ and $\mathrm{H} 4$, the detailed mechanisms underlying the loss of immunodominance of H60 remain unexplained.

In this study, to understand how the H60-specific CD8 $\mathrm{T}$-cell response becomes subservient to the $\mathrm{H} 4$-specific CD8 $\mathrm{T}$-cell response following allogeneic skin transplantation, we chased the immune dynamics of $\mathrm{H} 60$ and H4-specific CD8 $\mathrm{T}$ cells in $\mathrm{B} 6$ mice transplanted with BALB.B tail skin. The results demonstrate that the $\mathrm{H} 60$-specific $\mathrm{CD} 8 \mathrm{~T}$-cell response actively participates in the allo-response and that reliance of H60- or H4- specific CD8 $\mathrm{T}$ cells on the different antigen presentation pathways for dominance determines the immune hierarchy between the two antigens.

\section{MATERIALS AND METHODS}

\section{Mice}

C57BL/6 (B6: $\mathrm{H}-2^{\mathrm{b}}$ ) and C.B10-H2 $2^{b}$ LiMcdj (BALB.B: $\mathrm{H}-2^{\mathrm{b}}$ ) mice were purchased from the Jackson Laboratory (Bar Harbor, ME, USA). $\mathrm{H} 60$ congenic B6.C-H60 $/$ DCR and $\mathrm{H} 4$ congenic (B10.129- $H 46^{b} H 47^{b}$ $(21 \mathrm{M}) / \mathrm{Sn}$ ) mice were kindly provided by Dr Derry Roopenian ( The Jackson Laboratory). Luciferase transgenic mouse lines generated on $\mathrm{B} 6$ mice (B6-LucTg) were previously described, ${ }^{14}$ and maintained by crossing with $\mathrm{B} 6$ mice. All the mice were maintained at the Center for Animal Resource Development, Seoul National University College of Medicine, and all mouse experiments were performed in accordance with the guidelines and in compliance with the Institutional Animal Care and Use Committee (IACUC) of Seoul National University, Korea.

\section{Skin transplantation}

Skin was recovered and placed in PBS solution for a maximum of $30 \mathrm{~min}$ until use for transplantation. Full-thickness tail skin $\left(1 \times 1 \times 0.5 \mathrm{~cm}^{3}\right)$ derived from male BALB.B mice was transplanted on the tail site of C57BL/6J recipients. Recipient mice were anesthetized with evertin throughout the entire procedure. The skin graft was secured with a plastic adhesive bandage for 5 days. Graft survival was evaluated by daily visual inspection. Rejection was defined as necrosis of $75 \%$ of the transplanted skin surface.

\section{In vivo bioluminescence imaging (BLI) analysis}

In vivo bioluminescence imaging was performed using an IVIS 100 imaging system using a charge-coupled device camera (Caliper Life Sciences, Alameda, CA, USA). Mice were kept on the imaging stage under anesthesia with $1.5 \%$ isoflurane gas in oxygen at a flow rate of $1.51 \mathrm{~min}^{-1}$ and were given an i.p. injection of the substrate, D-luciferin (150 mg per $\mathrm{kg}$ body weight; Molecular Probes, Eugene, OR, USA). Mice were positioned supine to image the dorsal and ventral surfaces or on the left side to reveal the spleen or on the tail site. Relative intensities of emitted light were presented as pseudocolor images ranging from red (most intense) to blue (least intense). Gray-scale photographs and the corresponding pseudocolor images were superimposed with the LIVINGIMAGE (ver2.12; Xenogen, Alameda, CA, USA) and IGOR Pro (WaveMetrics, Lake Oswego, OR, USA) image analysis software. Signals emitted by regions of interest were measured and data were expressed as photon flux (photon $\mathrm{s}^{-1} \mathrm{~cm}^{-2}$ steradian-1 (sr-1)), which refers to the photons emitted from a unit solid angle of a sphere. Data are presented as the mean \pm

Figure 1 In vivo dynamics of CD8 T-cell expansion after allogeneic skin transplantation. (a) Experimental scheme of BLI analysis. CD8 T cells $\left(3 \times 10^{6}\right)$ isolated from female B6-LucTg mice were adoptively transferred into female B6-Albino mice one day prior to skin transplantation. Three pieces of male BALB.B skin and one piece of syngeneic female B6 skin were transplanted. (b) Graft survival in the transplanted B6-Albino mice. MHC-matched but minor $\mathrm{H}$ antigen-mismatched BALB.B skin $\left(\mathrm{H}-2^{\mathrm{b}}\right)$ were rejected $(n=18$, median survival time, MST = 19 days), whereas syngeneic B6 skin showed no signs of rejection ( $n=6$, MST $>40$ days). (c) Kinetics of bioluminescence intensity emitted by transferred B6-LucTg CD8 T cells after skin transplantation in the B6-Albino hosts. Bioluminescence imaging (BLI) was performed regularly and the in vivo imaging data demonstrate proliferation and migration of CD8 T cells in the hosts. Regions of interests (ROIs) were defined for the lumbar node as draining lymph node (dLN), grafted allogeneic BALB.B skin and syngeneic B6 skin, spleen and cervical lymph node as peripheral lymph node (PLN). Total photon outcomes from each ROI from three different mice were plotted along the time course. Data are presented as the mean \pm s.e.m. (d) Flow cytometric analysis of CD45.1 ${ }^{+}$CD8 $T$ cells in the adoptive CD45.2+ B6 hosts after skin transplantation. Cells present in the DLN, BALB.B skin graft, spleen and PLN of the transplanted mice were analyzed by flow cytometry at different time points after transplantation. Representative flow cytometric data are shown. Numbers of CD45.1 $1^{+}$CD4- cells were calculated and plotted. Data shown represent three (b) or two (c and d) independent experiments ( $n=3$ per group per experiment). 
standard error of the mean. The machine background was subtracted electronically, both from the images and from the measurements of photon flux.

\section{Antibodies and flow cytometry}

Peripheral blood leukocytes (PBLs), splenocytes and lymph node (LN) cells from recipient mice were incubated at $4{ }^{\circ} \mathrm{C}$ for $30 \mathrm{~min}$ in FACs
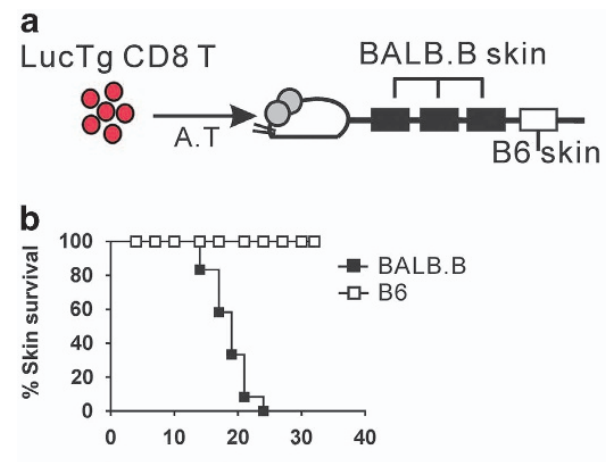

Days post-transplantation

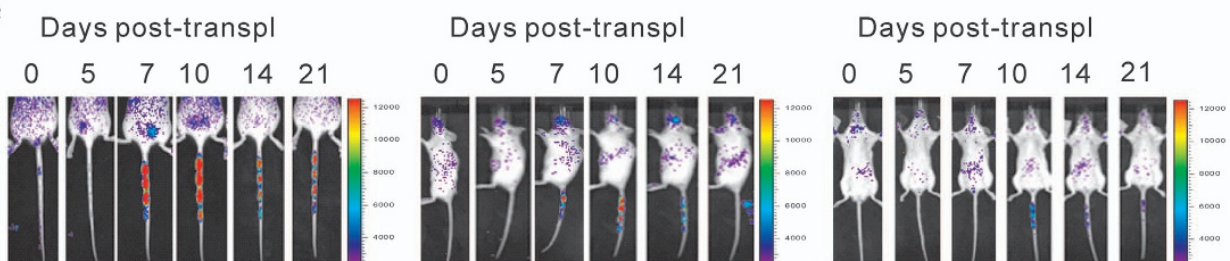

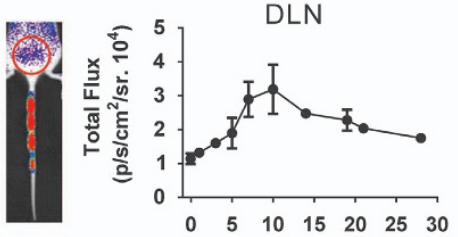

Days post-transplantation
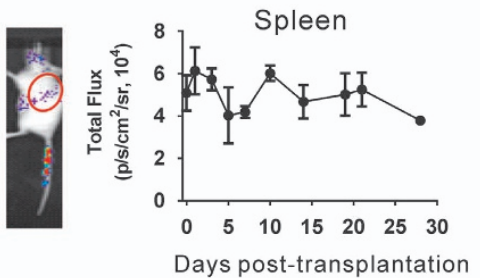
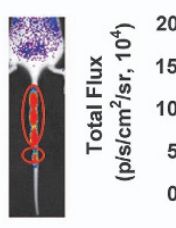

Skin Graft

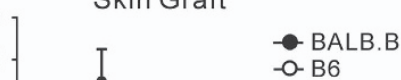

- B 6
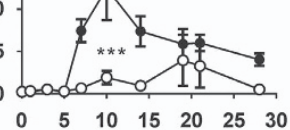

Days post-transplantation
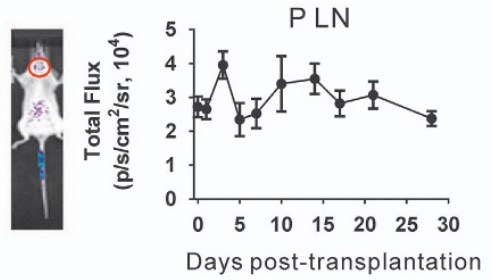

d

CD $45.1^{+}$CD8 T

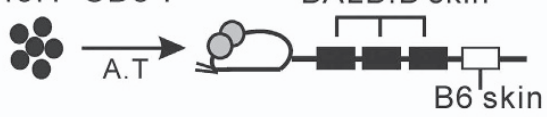

DLN Skin Graft Spleen $\frac{\text { PLN }}{\mathrm{O}^{10^{2}}}$

DLN-

Skin Graft
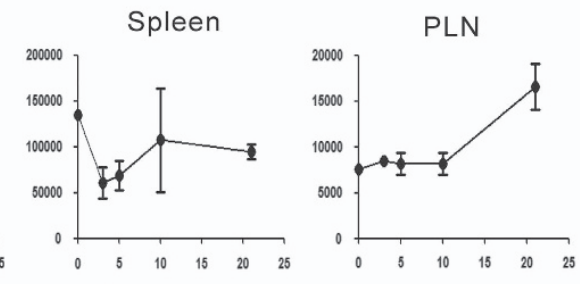

Days post-transplantation 
buffer $(1 \times$ PBS with $0.1 \%$ bovine calf serum and $0.05 \%$ sodium azide) containing phycoerythrin-labeled H60-peptide (LTFNYRNL) $/ \mathrm{H}-2 \mathrm{~K}^{\mathrm{b}}$ tetramer (H60-tetramer) or $\mathrm{H}^{\mathrm{b}}{ }^{\mathrm{b}}$-peptide (SGIVYIHL)/H-2K $\mathrm{K}^{\mathrm{b}}$ tetramer, and other fluorescence-labeled antibodies. The antibodies used for flow cytometry are anti-CD45.1, anti-CD11a, anti-CD8, anti-CD4 and anti-IFN- $\gamma$ mAbs purchased from eBioscience (San Diego, CA, USA).

\section{IFN- $\gamma$ secretion analysis}

Splenocytes and draining-LN cells-derived skin transplantation host cells $\left(1 \times 10^{7}\right.$ per well) were activated with $\mathrm{H} 60$ (LTFNYRNL), H4 ${ }^{\mathrm{b}}$ (SGIVYIHL) or VSV (RGYVYQGL) peptides $\left(10 \mu \mathrm{g} \mathrm{ml}^{-1}\right)$ for $2 \mathrm{~h}$ and treated with BFA for another $4 \mathrm{~h}$. Cells were washed twice with $1 \times$ PBS. After washing, activated cells were fixed with $1 \%$ PFA and stained with FITC-conjugated CD8 mAb, phycoerythrin-conjugated $\mathrm{H} 4 / \mathrm{H}-2 \mathrm{~K}^{\mathrm{b}}$ or $\mathrm{H} 60 / \mathrm{H}-2 \mathrm{~K}^{\mathrm{b}}$ tetramer and antigen-presenting cell (APC)conjugated IFN- $\gamma \mathrm{mAb}$.

\section{Statistical analysis}

Standard statistical methods were used to calculate the mean and standard error of the mean. The log-rank test was used to assess skingraft survival. Alternatively, Student's $t$-test was performed using the GraphPad Prism program version 4 (GraphPad Software, San Diego, CA, USA). A $P$ value $<0.05$ was considered to be statistically significant; ${ }^{\star} P<0.05,{ }^{* \star} P<0.01,{ }^{* * \star} P<0.001$.

\section{RESULTS}

\section{In vivo dynamics of $\mathrm{CD8} \mathrm{T}$-cell expansion after allogeneic skin transplantation}

To understand the in vivo dynamics of allo-antigen-reactive CD8 $\mathrm{T}$ cells during the allo-responses induced by allogeneic skin transplantation, we performed bioluminescence imaging analysis, which enables the non-invasive tracking of the migration and proliferation of cells in vivo. ${ }^{15}$ To this end, B6 background mice were transplanted with tail skin originating from MHC-matched but minor $\mathrm{H}$ antigen-mismatched BALB. $B$ mice. Female B6-Albino mice with white coat color, instead of wild-type (WT) black B6 mice, were chosen as recipients for the detection of BLI signals. One day prior to the skin transplantation, white B6-Albino mice were adoptively transferred with CD8 $\mathrm{T}$ cells isolated from luciferase transgenic mice (B6-LucTg), in which an enhanced firefly luciferase gene was expressed under the actin-promoter. ${ }^{14}$ These recipient mice were imaged periodically to obtain longitudinal bioluminescence images corresponding to the expansion and migration of the B6-LucTg CD8 T cells in the recipients until the rejection was completed (Figure 1a). Three pieces of the BALB.B tail skin were transplanted onto the tails of female B6-Albino mice, and a piece of female B6 skin was grafted behind the third BALB.B graft as a syngeneic control.

All three pieces of allogeneic BALB.B tail skin were completely rejected, with a median survival time of 19 days in the B6-Albino hosts (Figure 1b), as is usually observed with normal B6 hosts transplanted with BALB.B tail skin. The syngeneic graft of $\mathrm{B} 6$ tail skin remained accepted. The longitudinal BLI analysis showed that luminescence signals increased dramatically in the lumbar node from day 5 post transplantation; in comparison, increases in the signal in the spleens and other LNs located in the upper body (that is, cervical LNs) were not as high as that of the draining lumbar node (Figure 1c). This result implied that the increase in CD8 T-cell numbers was specific to the draining $\mathrm{LN}$ (LN) following allogeneic BALB.B skin transplantation. Strong luminescence signals were detected at the BALB.B allogeneic-skin graft from day 7 post transplantation, indicating the migration of activated $\mathrm{CD} 8 \mathrm{~T}$ cells to the allograft antigen sites. These signals lasted until the BALB.B allograft was rejected, with the peak signals being detected on day 10 post transplantation. The signals at the allograft sites remained at low intensities even after the rejection was complete (on days 21 and 28 post transplantation) and faded gradually (Figure 1c). Weak signals were also detected in syngeneic grafts, but only during the peak period (days 7-14). This was attributed to bystander signals generated by the high influx of allo-reactive CD8 $\mathrm{T}$ cells from the blood stream to the inflamed allograft sites.

To confirm the in vivo kinetics of CD8 T cells observed in BLI analysis, flow cytometric analyses were performed using B6 mice transplanted with BALB.B tail skin. CD $45.1^{+} \mathrm{CD} 8$ $\mathrm{T}$ cells were adoptively transferred into $\mathrm{CD} 45.2^{+} \mathrm{B} 6$ hosts one day prior to transplantation (Figure 1d), and their numbers in different organs of the transplanted hosts were estimated at different time points and plotted. Again, the CD45. $1^{+} \mathrm{CD} 8 \mathrm{~T}$-cell numbers increased dramatically in the dLNs and in the BALB.B allografts, with a slight increase observed in spleens. Consistent with the kinetics observed in the BLI analysis, the CD45.1 ${ }^{+} \mathrm{CD} 8 \mathrm{~T}$-cell numbers in the dLN and allograft peaked on day 10 post transplantation. Therefore, we concluded that the BLI data reflected the in vivo dynamics of $\mathrm{CD} 8 \mathrm{~T}$ cells following allogeneic skin transplantation.

\section{Transient immunodominance of $\mathrm{H60}$ prior to overall prominent dominance of $\mathrm{H} 4$ during anti-BALB.B allo- response}

Next, we tested whether the immune hierarchy (H4> H60) could be reproduced in our tail skin transplantation experiment. Leukocytes in blood, spleen and draining LN were stained with $\mathrm{H} 60$-and H4-tetramers and analyzed via flow cytometry at three different time points, days 7,10 and 14 post transplantation. The results showed that frequencies of H4-tetramer-binding CD8 T cells were exceptionally high, with a peak range of $6-16 \%$ of blood and spleen CD8 T cells (12.3\% on average) on day 10 post transplantation, demonstrating that the H4-specific CD8 T-cell response was dominant overall (Figure 2a). However, unexpectedly, we observed that the frequencies of H60-tetramer-binding CD8 $\mathrm{T}$ cells in the blood $(4.88 \%$ and $3.22 \%$ in PBL and spleen CD8 T cells, respectively, on average) were higher than those of H4-tetramer-binding cells $(1.47 \%$ and $1.88 \%$, respectively on average) on day 7 . This indicates that there was a transient period of $\mathrm{H} 60$ dominance before $\mathrm{H} 4$ dominance became prominent. This trend of differential kinetics between $\mathrm{H} 60$ - and $\mathrm{H} 4$-specific $\mathrm{CD} 8 \mathrm{~T}$-cell responses was 
a
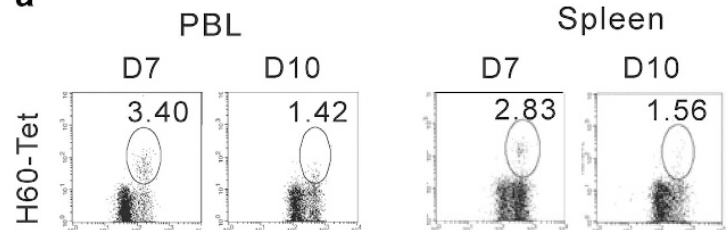

DLN
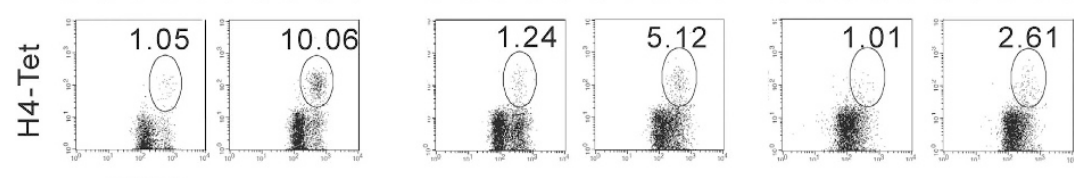

CD11a
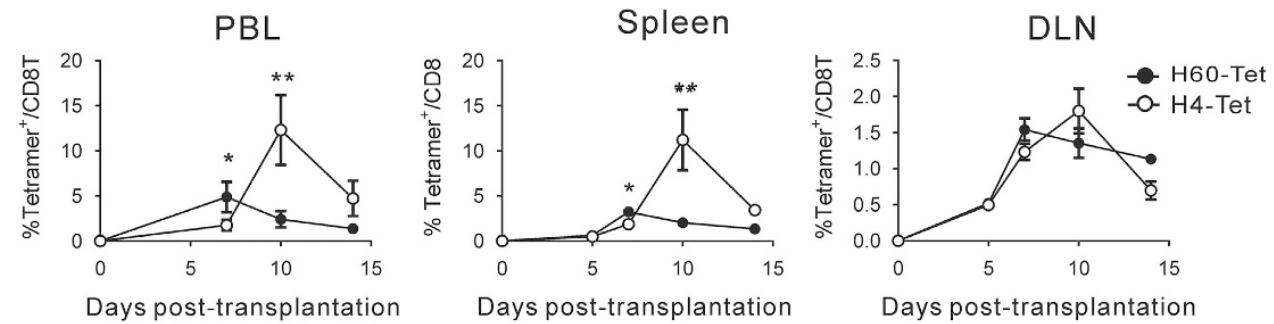

b

Days post-transplantation

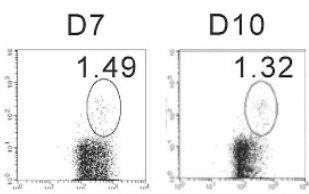

Days post-transplantation

Days post-transplantation
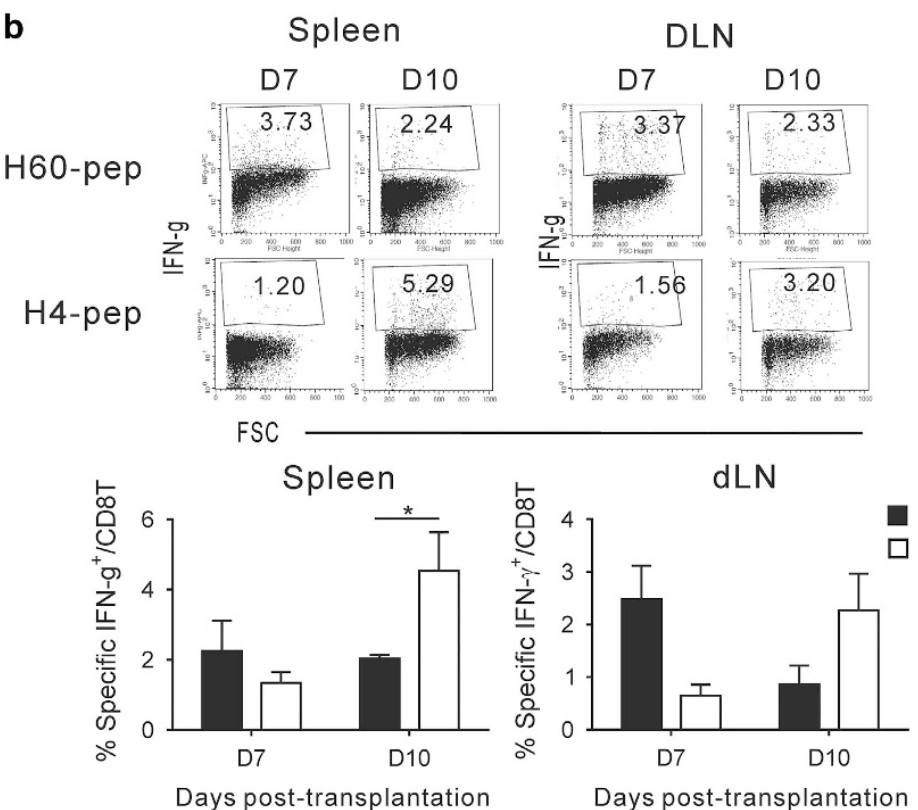

H60-stimulation

H4-stimulation

Figure 2 Transient immunodominance of $\mathrm{H} 60$ prior to overall prominent dominance of $\mathrm{H} 4$ during anti-BALB.B allo-response. (a) Flow cytometric analysis of PBLs, splenocytes and draining LN cells in female B6 mice at different time points after BALB.B skin transplantation. Cells were stained with $\mathrm{H} 60$ - and $\mathrm{H} 4$-tetramers, in combination with anti-CD8 and anti-CD11b monoclonal antibodies. Data were analyzed after gating for $\mathrm{CD}^{+}$cells. Percentages of each tetramer-binding cells among $\mathrm{CD} 8^{+}$cells were denoted in the flow cytometric data and the values were plotted. Representative flow cytometric data obtained on days 7 and 10 are shown. (b) IFN- $\gamma$-secretion analysis of CD8 T cells in spleens and draining LN on days 7 and 10 post transplantation. CD8 T cells were analyzed for IFN- $\gamma$ production in response to stimulation with $\mathrm{H60}, \mathrm{H} 4$ or VSV peptide. Representative flow cytometric data are shown. Percentage values were deduced of values obtained after VSV-stimulation and were plotted. Data shown represent two independent experiments ( $n=3$ per group per experiment).

confirmed by IFN- $\gamma$ secretion analysis using the CD8 T cells present in the spleen and dLN (Figure 2b). This transient weak dominance of $\mathrm{H} 60$, in spite of the overall dominance of $\mathrm{H} 4$, implied that the H60-specific CD8 $\mathrm{T}$ cells were not dormant during the anti-BALB.B skin allo-response but demonstrated that they participated actively in the response, particularly early after transplantation.
Participation of $\mathrm{H60}$-specific CD8 $\mathrm{T}$ cells in the allo-response after skin transplantation

On the basis of our findings that H60-specific CD8 T cells dominate early and briefly, we sought to investigate their dynamics during the allo-response against BALB.B skin. In particular, we questioned whether they could migrate to the BALB.B allografts in which parenchymal cells do not express 
H60. To address this question, we performed BLI analysis on CD8 T cells specific for H60 in the BALB.B skin-transplanted hosts using B6-Albino mice that had been adoptively transferred with CD8 $\mathrm{T}$ cells expressing transgenic luciferase and TCRs (J15) with specificity to H60 before skin transplantation (Figure 3a). Transgenic TCR mouse lines were generated on the B6 background, using the TCR $\alpha$ and $\beta$ sequences obtained from a previously described $\mathrm{H} 60$-specific CTL clone that recognizes the $\mathrm{H} 60$-epitope $/ \mathrm{H}-2 \mathrm{~K}^{\mathrm{b}}$ with moderate affinity. ${ }^{16}$ Among the three different lines expressing the transgenic TCRs, a transgenic mouse line named J15 expressed TCRs on CD8 T cells at levels comparable with those of normal TCRs on polyclonal B6 CD8 T cells, as determined by flow cytometric analysis (data not shown). The J15 line was therefore chosen for crossing with B6-LucTg mice to generate double transgenic mice.

BLI analysis of J15-LucTg CD8 T cells in the transplanted hosts visualized the expansion of those in the draining $\mathrm{LN}$ and migration to the BALB.B allograft (Figure 3b). To verify the specific migration of H60-specific CD8 T cells to the allograft whose parenchymal cells do not express H60, another group of adoptive B6-Albino hosts were transplanted with skin from H60 congenic mice. The BLI data showed migration of the H60-specific CD8 T cells to the grafted H60 congenic skin. However, the signal kinetics curve demonstrated that the expansion and migration of the H60-specific CD8 T cells were slightly delayed in the host transplanted with $\mathrm{H} 60$ congenic skin, compared with those with BALB.B skin. This result indicated that responses of H60-specific CD8 $\mathrm{T}$ cells were influenced by the presence or absence of immune competition with CD8 $\mathrm{T}$ cells specific for $\mathrm{H} 4$ and other minor $\mathrm{H}$ antigens. Nevertheless, it was clear that the H60-specific CD8 T cells could migrate to the allografts, despite the hematopoietic cellrestricted distribution of $\mathrm{H} 60$.

Next, using flow cytometric analysis with B6 mice transplanted with the two different sources of tail skin, we confirmed the difference in the response kinetics of $\mathrm{H} 60$ specific CD8 T cells between BALB.B and H60 congenic skin transplantation. B6 mice transplanted with $\mathrm{H} 4$ congenic skin were included as a control group. Compared with the rejection of BALB.B and H4 congenic skin (17 and 19 days of median survival times, respectively; Figure 3c), the transplanted H60 congenic skin was rejected, with a slight, albeit insignificant, delay (24 days of median survival time). H60-tetramer-binding CD8 $\mathrm{T}$ cells were detected in the blood of the hosts transplanted with H60 congenic skin, with delayed peak days (3.4\% on average at day 10 ) compared with that after BALB.B skin transplantation (average $3.5 \%$ at day 7 ). Thus, response of H60-specific CD8 $\mathrm{T}$ cells was accelerated in the presence of immune competition. On the contrary, the H4-specific CD8 $\mathrm{T}$-cell response was relatively stable, reaching its peak on the same day, regardless of the presence (BALB.B skin transplantation) or absence ( $\mathrm{H} 4$ congenic skin transplantation) of immune competition. Therefore, the $\mathrm{H} 60$-specific response was relatively dynamic and was influenced by immune competition.
Reversed immune hierarchy by the enhancement of H60specific CD8 T-cell frequency

Because BLI and flow cytometric data provided evidence supporting an active involvement of H60-specific CD8 T cells in allo-responses against BALB.B skin grafts, we tested whether the enhancement of H60-specific CD8 T-cell frequency prior to allogeneic skin transplantation could influence the rejection process and immune hierarchy between $\mathrm{H} 60$ and $\mathrm{H} 4$. To enhance the frequency, female B6 mice were pre-immunized with male H60 congenic splenocytes 30 days prior, and then transplanted with BALB.B skin. The frequency of H4-specific CD8 $\mathrm{T}$ cells before BALB.B skin transplantation was enhanced in the same way, by immunizing B6 female mice with $\mathrm{H} 4$ congenic splenocytes in parallel. Therefore, the frequencies of $\mathrm{H} 60$ or $\mathrm{H} 4$-specific CD8 T cells were comparable, being in the range of $0.2-0.3 \%$ among blood CD8 $\mathrm{T}$ cells of the respective pre-immune mice one day prior to the BALB.B skin transplantation (data not shown). Upon transplantation, BALB.B skin was rejected more rapidly in the hosts pre-immunized against either $\mathrm{H} 60$ or $\mathrm{H} 4$ (with 12 or 14 days of median survival time, respectively) than in hosts without pre-immunization (17 days of MTS; Figure 4a). Therefore, regardless of whether the specificity was for hematopoietic cell specific $\mathrm{H} 60$ or ubiquitous $\mathrm{H} 4$ antigen, the presence of memory CD8 $\mathrm{T}$ cells in the hosts expedited the rejection of the allogeneic BALB.B skin, verifying the involvement of H60-specific CD8 $\mathrm{T}$ cells in the anti-BALB.B skin response.

As expected, flow cytometric analysis of PBLs revealed that pre-immunization with $\mathrm{H} 60$ enhanced the frequencies of the specific CD8 T cells detected after BALB.B skin transplantation. This led to significant reductions in the frequencies of the H4specific CD8 T cells in blood, compared with that in hosts without $\mathrm{H} 4$ pre-immunization (Figure $4 \mathrm{~b}$ ). This demonstrated a reversion of the immune hierarchy between $\mathrm{H} 60$ and H4, because of formation of memory cells specific for $\mathrm{H} 60$ before BALB.B skin transplantation. Pre-immunization for $\mathrm{H} 4$ also increased H4-reactive CD8 T-cell frequencies slightly, but not significantly. Altogether, these results implied that although the H60-specific response was subservient to the H4-specific CD8 T-cell response overall, H60 was not an insignificant antigen but an influential one during the development of anti-BALB.B skin allo-responses.

\section{Dependence of $\mathrm{H60}$ dominance on direct antigen presentation by donor APCs}

After allogeneic skin transplantation, direct presentation by donor APCs and indirect presentation by host APCs are known to contribute to the activation of all-reactive T cells. ${ }^{17,18} \mathrm{We}$ investigated the extents by which these pathways contributed to the induction of the CD8 T-cell responses for $\mathrm{H} 60$ and H4, with the hypothesis that differential distributions of these two antigens would influence the antigen presentation pathway. To evaluate the contribution of direct presentation by donor APCs, we used $\beta 2 \mathrm{~m}$-deficient mice as recipients of the BALB. B skin transplantation, which were adoptively transferred with 
a

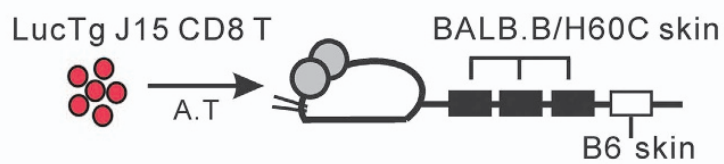

b

BALB.B skin

H60C skin

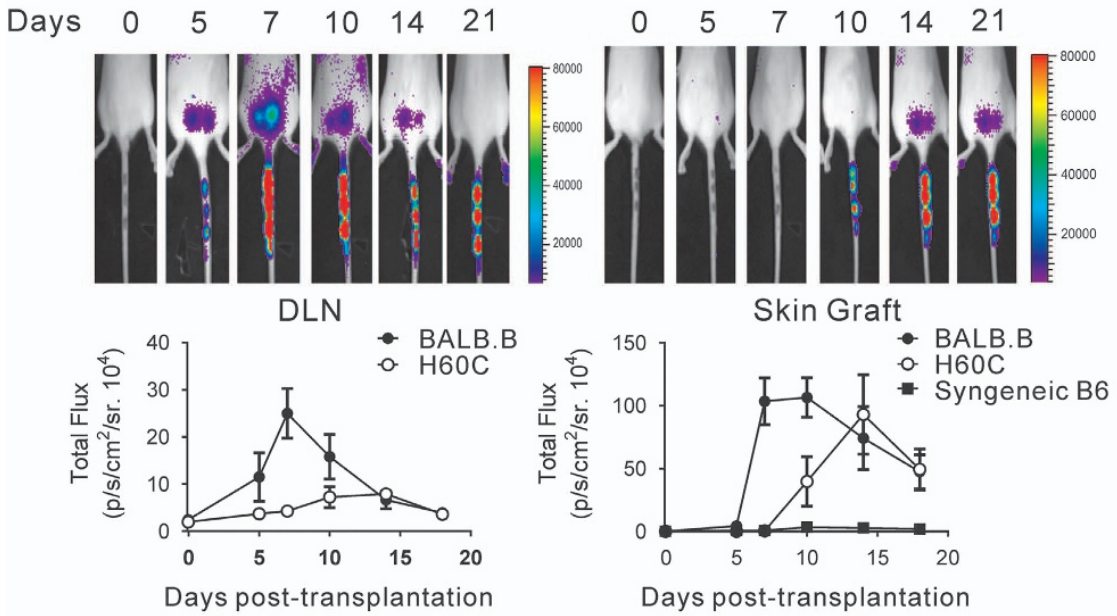

C

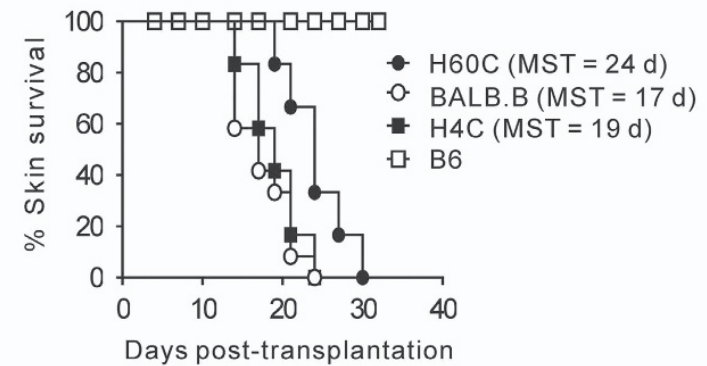

d
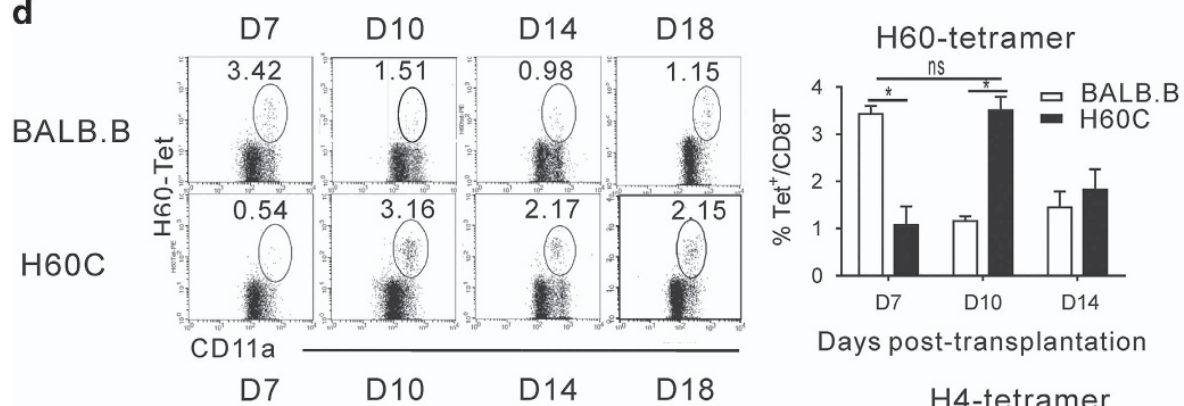

H60C

BALB.B

Days post-transplantation

BALB.B
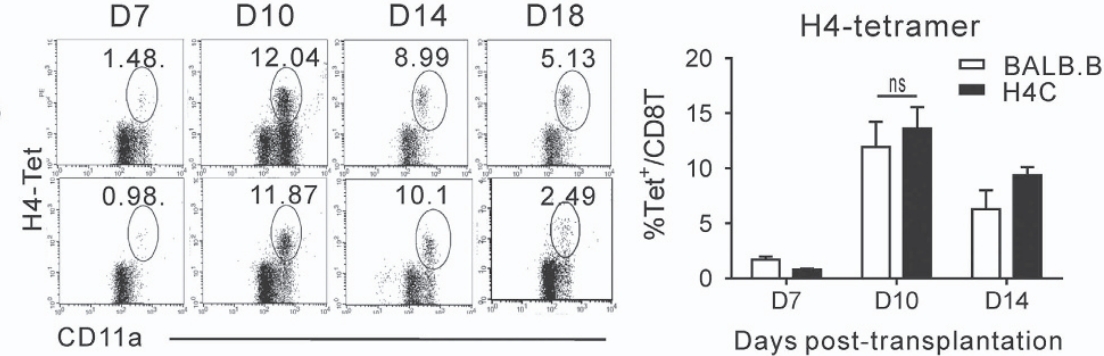

Figure 3 Participation of H60-specific CD8 T cells in the allo-response after skin transplantation. (a) Experimental scheme of BLI analysis on H60-specific CD8 T cells after BALB.B skin or H60 congenic skin transplantation. B6-LucTg J15 CD8 T cells $\left(1 \times 10^{5}\right)$ were adoptively transferred to B6-Albino mice one day prior to BALB.B skin transplantation. (b) J15 CD8 T cells were monitored periodically after transplantation of BALB.B skin or H60 congenic skin onto B6 mice. Imaging data shown represent two independent experiments ( $n=3$ per experiment). Total flux values obtained from the draining LNs and grafted allogeneic or syngeneic skin were plotted. (c-d) Responses of female B6 mice against different allo-grafts from BALB.B, H60 congenic or H4 congenic mice. (c) Survival of the different grafts. (d) Longitudinal flow cytometric analysis of peripheral blood leukocytes after transplantation. Representative flow cytometric data after gating for $\mathrm{CD}^{+}$cells are shown. Frequencies of H60-tetramer and H4-tetramer-binding CD8 T cells in blood are plotted. Data shown represent two independent experiments ( $n=3$ per group per experiment). 


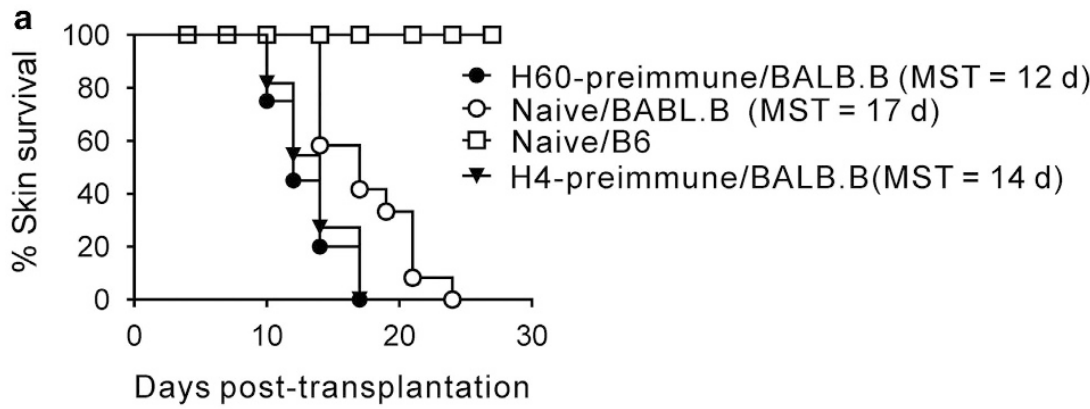

b

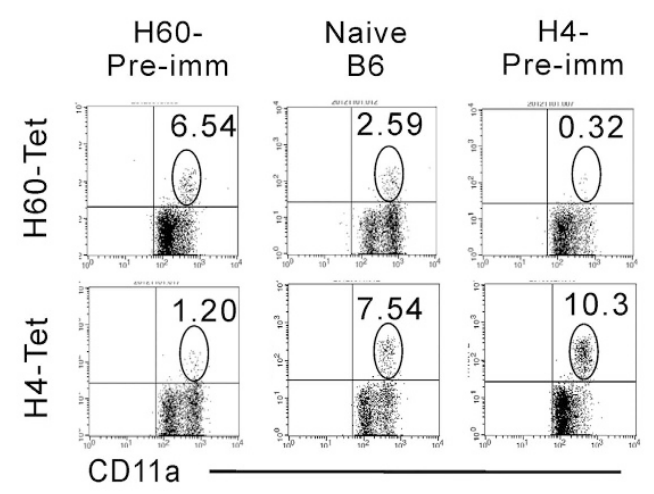

H60-Tetramer

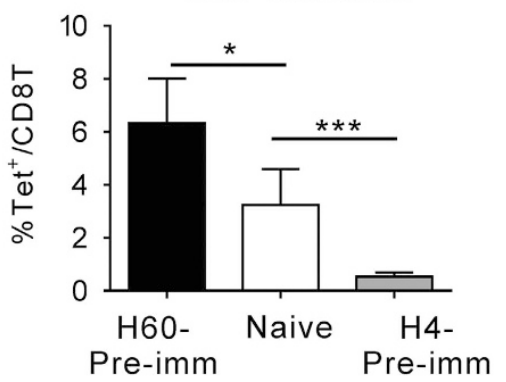

H4-Tetrmaer

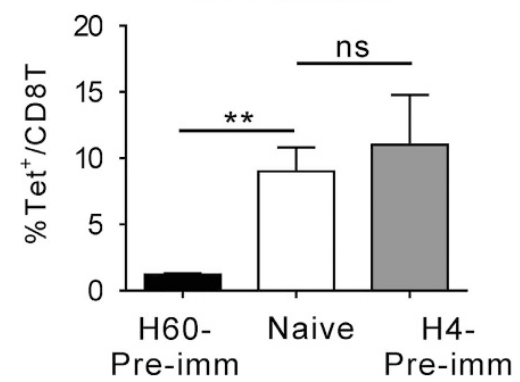

Figure 4 Reversed immune hierarchy by the enhancement of H60-specific CD8 T-cell frequency. (a) Survival of grafted BALB.B skin in B6 mice under different immune conditions. Naïve female B6 mice or B6 mice that were pre-immunized with splenocytes $\left(2 \times 10^{7}\right)$ from male $\mathrm{H} 60$ congenic or $\mathrm{H} 4$ congenic mice 30 days prior were transplanted with BALB.B skin. Survival of BALB.B grafts collected from two independent experiments were plotted ( $n=12$ per group). (b) Flow cytometric analysis of peripheral blood leukocytes after staining with H60- or H4-tetramers. Representative data obtained on day 10 post transplantation of BALB.B skin are shown. Frequencies of tetramerbinding CD8 T cells are plotted. Two independent experiments were performed ( $n=3$ per group per experiment).

CD45.1 $1^{+}$WT CD8 $\mathrm{T}$ cells 3 weeks prior; in this way, the activation of CD8 $\mathrm{T}$ cells would necessarily rely on antigen presentation by donor APCs present in the grafted BALB.B skin. The transferred CD45.1 ${ }^{+}$WT CD8 T cells were stabilized for 3 weeks in the $\beta 2 \mathrm{~m}$-deficient hosts before induction of the allo-response (Figure 5a). The $\beta 2 \mathrm{~m}$-deficient hosts were transplanted with skin from $\mathrm{H} 60$ congenic or $\mathrm{H} 4$ congenic mice in parallel.

In flow cytometric analysis of PBLs from the transplanted $\beta 2 \mathrm{~m}$-deficient hosts, H60-tetramer-binding CD8 T cells were detected. However, the total CD45.1 $1^{+}$CD8 $\mathrm{T}$ cells were less abundant than in normal mice, and the peak frequencies were significantly enhanced to $5.6 \%$ on average, compared with the values obtained with BALB.B skin transplanted WT counterparts on day 7 post transplantation (Figures $5 \mathrm{~b}$ and c). These values were also significantly higher than those detected after H60 congenic skin transplantation onto $\beta 2 \mathrm{~m}$ deficient hosts. Therefore, the presence of immune competition and direct presentation only encouraged the expansion of the H60-specific CD8 T cells after BALB.B skin transplantation. On the contrary, H4-tetramer-binding $\mathrm{CD} 8 \mathrm{~T}$ cells were rarely detected in the $\beta 2 \mathrm{~m}$-deficient hosts transplanted with BALB.B or $\mathrm{H} 4$ congenic skin (Figures $5 \mathrm{~b}$ and $\mathrm{c}$ ), indicating the complete abrogation of H4-immunodominance in the absence of indirect presentation. These results suggested that immunodominance of $\mathrm{H} 60$ might rely on direct antigen presentation by donor APCs, whereas that of $\mathrm{H} 4$ might rely on indirect presentation by host APCs. 
a
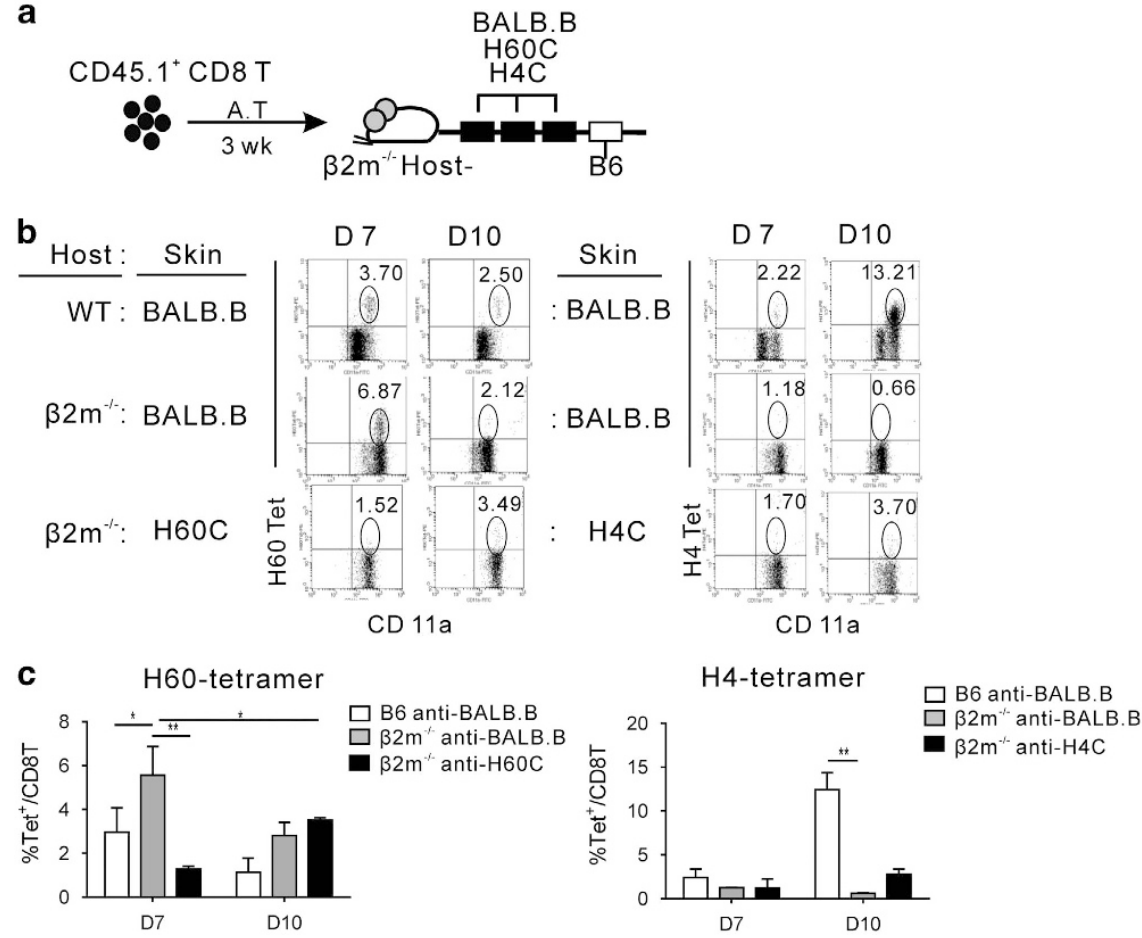

Figure 5 Dependence of $\mathrm{H} 60$ dominance on direct antigen presentation by donor APCs. (a) Experimental scheme. CD45. $1^{+}$CD8 $\mathrm{T}$ cells were adoptively transferred into $\beta 2 \mathrm{~m}^{-/}$mice which were then transplanted with skin from BALB.B, H60 or H4 congenic mice 3 weeks later. B6 control mice were transplanted with BALB.B skin. (b) peripheral blood leukocyte (PBL) analysis of the transplanted mice. PBLs from the transplanted mice were analyzed by flow cytometry after staining with H60-tetramers and H4-tetramers. Representative data after gating on $\mathrm{CD}^{+}$cells are shown. Frequencies of the tetramer-binding CD8 T cells in blood were plotted. Data shown (b, c) represent two independent experiments ( $n=3$ per group per experiment).

\section{Lack of full expansion of H60-specific CD8 T cells in the absence of direct presentation after allogeneic skin transplantation.}

Next, we examined whether the lack of direct presentation would affect the magnitude of H60-specific CD8 T-cell responses after skin transplantation. To this end, we transplanted B6 mice with skin from $\mathrm{H} 60$ congenic mice crossed onto the $\beta 2 \mathrm{~m}$-deficient background $\left(\beta 2 \mathrm{~m}^{-/} \mathrm{H} 60\right.$ congenic mice); in this way, donor APCs originating from the $\beta 2 \mathrm{~m}$ deficient $\mathrm{H} 60$ congenic mice could not present $\mathrm{H} 60$, and consequently, the activation of H60-specific CD8 T cells would necessarily rely on indirect presentation by host APCs (Figure 6a). As controls, skin from $\mathrm{H} 4$ congenic mice on the $\beta 2 \mathrm{~m}$-deficient background $\left(\beta 2 \mathrm{~m}^{-/-} \mathrm{H} 4\right.$ congenic mice) or BALB.B mice was transplanted onto WT B6 mice in parallel. In flow cytometric PBL analysis, H60-tetramer-binding CD8 $\mathrm{T}$ cells were rarely detected in the blood of the host transplanted with $\beta 2 \mathrm{~m}$-deficient $\mathrm{H} 60$ congenic skin (Figure 6b). However, H4-tetramer-binding CD8 T cells were detected at significant frequencies in the blood of mice transplanted with $\beta 2 \mathrm{~m}$-deficient $\mathrm{H} 4$ skin. However, these frequencies were slightly, albeit not significantly, lower than the usual values observed with WT H4 congenic skin, demonstrating that both indirect and direct antigen presentation pathways contributed to full expansion of $\mathrm{H} 4$-specific CD8 $\mathrm{T}$ cells after skin transplantation. However, the indirect pathway only could support a normal range of expansion of H4-specific CD8 T cells, but not that of H60-specific CD8 $\mathrm{T}$ cells under skin transplantation conditions. Therefore, the immunodominance of $\mathrm{H} 60$ after skin transplantation was dependent on the direct antigen presentation pathway, whereas that of $\mathrm{H} 4$ was more dependent on the indirect pathway.

\section{DISCUSSION}

In this study, we demonstrate that CD8 T-cell response against the hematopoietic lineage-specific antigen $\mathrm{H} 60$ participates in the allo-response induced after BALB.B skin transplantation and migrates to the grafted BALB.B skin, although this response is subdominant to the H4-specific CD8 T-cell response overall. However, we discovered that before the H4immunodominance becomes prominent, there is a transient period during which $\mathrm{H} 60$ is slightly immunodominant. This dynamic change in dominance between $\mathrm{H} 60$ and $\mathrm{H} 4$ is related to differences in preferences for antigen presentation pathways for each of these antigens during the anti-BALB.B skin alloresponse. The reliance on the direct presentation pathway is suggested to be the reason for the transient dominance and overall subdominance of the H60-specific CD8 T-cell response after BALB.B skin transplantation.

Immunodominance of $\mathrm{H} 60$ during the $\mathrm{B} 6$ anti-BALB.B response has been established under circumstances, in which the transplanted materials involved hematopoietic cells, 


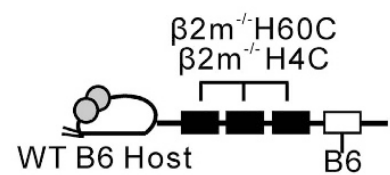

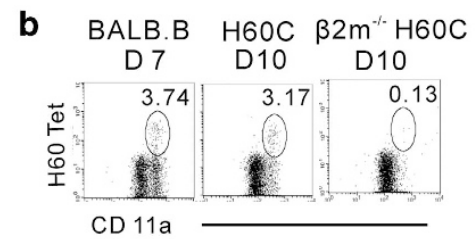

H60-tetramer

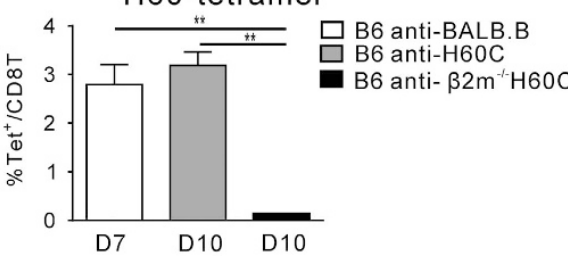

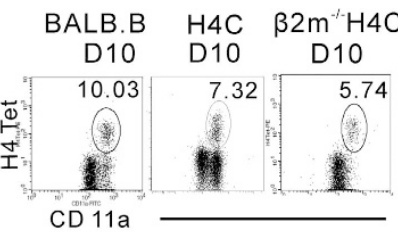

H4-tetramer

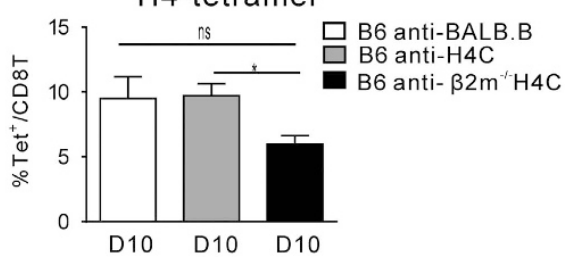

Figure 6 Lack of full expansion of H60-specific CD8 T cells in the absence of direct presentation after allogeneic skin transplantation. (a) Experimental scheme. WT B6 mice were transplanted with $\beta 2 \mathrm{~m}^{-/-} \mathrm{H} 60$ congenic or $\beta 2 \mathrm{~m}^{-1-} \mathrm{H} 4$ congenic skin. Control mice were transplanted with BALB.B, H60 congenic or H4 congenic skin. (b) PBLs of the transplanted mice were analyzed by flow cytometry after staining with $\mathrm{H} 60$-tetramers and H4-tetramers. Representative data obtained after gating on $\mathrm{CD}^{+}$cells are shown. Frequencies of the tetramer-binding CD8 T cells in blood were plotted. Data shown (b) represent two independent experiments ( $n=3$ per group per experiment).

such as spleen cell immunization, graft-versus-host disease induction and heart transplantation involving primary vascularization. ${ }^{6,8,11,13}$ Consistent with a previous report, we demonstrated that the H60-specific response was subdominant to the H4-specific response after BALB.B tail skin transplantation. ${ }^{13}$ One interpretation of this dominant H4specific response is that CD8 T-cell responses specific for the hematopoietic cell-restricted antigen H60 plays an insignificant role in solid tissue transplantation, which does not involve vascularization. However, our findings indicated that H60-specific CD8 T cells actively participate in the anti-BALB. $\mathrm{B}$ skin allo-response because (i) the $\mathrm{H} 60$-specific response was not consistently subdominant, but was instead briefly dominant, and (ii) pre-immunization with $\mathrm{H} 60$ reversed the immune hierarchy and expedited the rejection of BALB.B skin. Thus, the immunodominance of $\mathrm{H} 4$ after BALB.B skin transplantation is neither stable nor permanent, but can be subdominant, especially when antigen presentation pathways are manipulated. We further suggest that the immunodominance of a specific antigen may reflect the ongoing immune responses during the rejection process after allograft transplantation.

The fact that dominance of the H60-specific CD8 T-cell response emerged briefly before the $\mathrm{H} 4$-specific CD8 T-cell response predominated suggested that the expansion of $\mathrm{H} 60$ specific CD8 $\mathrm{T}$ cells in response to BALB.B skin transplantation would precede that of H4-specific CD8 T cells. During the rejection process after solid tissue transplantation, donor APCs leave the grafted tissue and reach the draining LN, where they activate T cells. ${ }^{19}$ Among the donor APCs in the skin, dermal dendritic cells were found to disappear from the graft within 3-5 days, constituting the first wave of APCs reaching the draining LN, whereas Langerhans cells persisted until the onset of graft rejection. ${ }^{20}$ Langerhans cells have been suggested to play a role at later time points or cause additional activation of $\mathrm{T}$ cells, rather than priming $\mathrm{T}$ cells. ${ }^{21}$ Combining these with our results, we suggest that H60-specific CD8 $\mathrm{T}$ cells may first be primed by donor dermal dendritic cells in the $\mathrm{dLN}$, and then infiltrate to the allografts where they are further stimulated by long-lasting Langerhans cells in the grafted BALB.B tissues. However, because of the low numbers of H60-expressing Langerhans cells in the grafted allogeneic skin, further expansion and participation of H60-specific CD8 $\mathrm{T}$ cells in allo-response may be limited, eventually becoming subservient to $\mathrm{H} 4$ specific CD8 T cells.

H4 dominance was found to depend more on the indirect rather than the direct presentation pathway. Sources of donor allo-antigens for indirect presentation include donor cells that migrate and die in the draining LN. ${ }^{22}$ Alternatively, host APCs, such as inflammatory monocytes and dendritic cells, which infiltrate the allograft, may collect antigens in the grafts and migrate to the draining $\mathrm{LN}$, where they cross-prime CD8 $\mathrm{T}$ cells. ${ }^{20,23}$ Considering the later emergence of H4-reactive CD8 $\mathrm{T}$ cells than H60-specific cells in the blood, we suggest that the indirect antigen presentation by host APCs infiltrating the allografts would be responsible for the overall dominance of the H4-specific CD8 T-cell response. Being expressed by various cell types, H4 is relatively abundant in the skin grafts, allowing the indirect pathway to support the high magnitude of H4-specific CD8 T-cell expansion. Therefore, the distribution of an alloantigen influences antigen presentation pathways that 
lead to the dominance of specific CD8 T-cell responses, thereby, determining the immune hierarchy after allogeneic skin transplantation. Although the H60-specific CD8 T-cell expansion was dependent on the direct pathway under skin transplantation conditions, in the cases of allogeneic hematopoietic cell transplantation (such as spleen cell immunization), the massive expansion of H60-specific CD8 T cells was also more dependent on indirect presentation by host APCs than direct presentation by donor immunizing cells. ${ }^{24,25}$ Taking all of these findings obtained under different experimental conditions, we propose that regardless of whether the stimulating sources are hematopoietic cells or non-hematopoietic solid tissues, the indirect antigen presentation pathway would contribute more to immunodominance than the direct pathway.

Mechanisms of immunodominance have been investigated intensively in the responses following allogeneic transplantation and pathogen infections. ${ }^{26}$ The determining factors vary but may include affinity of peptide/MHC complexes for the specific TCRs, competition for target cells and frequencies of reactive CD8 $\mathrm{T}$ cells. ${ }^{11,27-30}$ Here, we demonstrate that immunodominance is influenced by the alloantigendistribution pattern and associated antigen presentation pathway.

In summary, we demonstrated that although the H60specific CD8 $\mathrm{T}$ cells actively participate in the allo-response induced by BALB.B skin transplantation, the specific response is transiently dominant, but is overall subdominant to the H4-specific CD8 T-cell response. Moreover, the dependence on different antigen presentation pathways for the immunodominance between $\mathrm{H} 60$ and $\mathrm{H} 4$ leads to the immune hierarchy of $\mathrm{H} 60<\mathrm{H} 4$ during the anti-BALB.B allo-response. Our results not only enhance the understanding of the immunodominance phenomenon but also provide useful information for controlling immune response after allogeneic tissue transplantation.

\section{ACKNOWLEDGEMENTS}

This study was supported by a grant from the Korea Healthcare Technology R \& D project, Ministry of Health, Welfare, and Family Affairs of Korea (HT 12C0223).

1 Wallny HJ, Rammensee HG. Identification of classical minor histocompatibility antigen as cell-derived peptide. Nature 1990; 343 : 275-278.

2 Roopenian D, Choi EY, Brown A. The immunogenomics of minor histocompatibility antigens. Immunol Rev 2002; 190: 86-94.

3 Teh HS, Bennink J, Von Boehmer H. Selection of the $T$ cell repertoire during ontogeny: limiting dilution analysis. Eur J Immunol 1982; 12: 887-892.

4 Perreault C, Roy DC, Fortin C. Immunodominant minor histocompatibility antigens: the major ones. Immunol Today 1998; 19: 69-74.

5 Wettstein PJ, Bailey DW. Immunodominance in the immune response to "multiple" histocompatibility antigens. Immunogenetics 1982; 16: 47-58.

6 Choi EY, Yoshimura Y, Christianson GJ, Sproule TJ, Malarkannan S, Shastr $\mathrm{N}$ et al. Quantitative analysis of the immune response to mouse non-MHC transplantation antigens in vivo: the $\mathrm{H} 60$ histocompatibility antigen dominates over all others. J Immunol 2001; 166: 4370-4379.

7 Anderson CC, Matzinger P. Immunity or tolerance: opposite outcomes of microchimerism from skin grafts. Nat Med 2001; 7: 80-87.

8 Choi EY, Christianson GJ, Yoshimura Y, Jung N, Sproule TJ, Malarkannan S et al. Real-time T-cell profiling identifies $\mathrm{H} 60$ as a major minor histocompatibility antigen in murine graft-versus-host disease. Blood 2002; 100 : 4259-4265.

9 Wettstein PJ, Colombo MP. Immunodominance in the T cell response to multiple non-H-2 histocompatibility antigens. IV. Partial tissue distribution and mapping of immunodominant antigens. J Immunol 1987; 139: 2166-2171.

10 Malarkannan S, Shih PP, Eden PA, Horng T, Zuberi AR, Christianson G et al. The molecular and functional characterization of a dominant minor $\mathrm{H}$ antigen, H60. J Immunol 1998; 161: 3501-3509.

11 Choi EY, Christianson GJ, Yoshimura Y, Sproule TJ, Jung N, Joyce S et al. Immunodominance of $\mathrm{H} 60$ is caused by an abnormally high precursor T cell pool directed against its unique minor histocompatibility antigen peptide. Immunity 2002; 17: 593-603.

12 Yadav R, Yoshimura Y, Boesteanu A, Christianson GJ, Ajayi WU, Shashidharamurthy $\mathrm{R}$ et al. The $\mathrm{H} 4 \mathrm{~b}$ minor histocompatibility antigen is caused by a combination of genetically determined and posttranslational modifications. J Immunol 2003; 170: 5133-5142.

13 Kwun J, Malarkannan S, Burlingham WJ, Knechtle SJ. Primary vascularization of the graft determines the immunodominance of murine minor $\mathrm{H}$ antigens during organ transplantation. J Immunol 2011; 187: 3997-4006.

14 Song MG, Kang B, Jeon JY, Chang J, Lee S, Min CK et al. In vivo imaging of differences in early donor cell proliferation in graft-versus-host disease hosts with different pre-conditioning doses. Mol cells 2012; 33: 79-86.

15 Negrin RS, Contag $\mathrm{CH}$. In vivo imaging using bioluminescence: a tool for probing graft-versus-host disease. Nat Rev Immunol 2006; 6: 484-490.

16 Jeon JY, Jung KM, Chang J, Choi EY. Characterization of CTL clones specific for single antigen, $\mathrm{H} 60$ minor histocompatibility antigen. Immune Netw 2011; 11: 100-106.

17 Ochando JC, Krieger NR, Bromberg JS. Direct versus indirect allorecognition: Visualization of dendritic cell distribution and interactions during rejection and tolerization. Am J Transplant 2006; 6: 2488-2496.

18 Valujskikh A, Hartig C, Heeger PS. Indirectly primed CD8+ T cells are a prominent component of the allogeneic T-cell repertoire after skin graft rejection in mice. Transplantation 2001; 71: 418-421.

19 Larsen CP, Austyn JM, Morris PJ. The role of graftderived dendritic leukocytes in the rejection of vascularized organ allografts. Recent findings on the migration and function of dendritic leukocytes after transplantation. Ann Surg 1990; 212: 308-315 discussion 316-317.

20 Celli S, Albert ML, Bousso P. Visualizing the innate and adaptive immune responses underlying allograft rejection by two-photon microscopy. Nat Med 2011; 17: 744-749.

21 Obhrai JS, Oberbarnscheidt M, Zhang N, Mueller DL, Shlomchik WD, Lakkis FG et al. Langerhans cells are not required for efficient skin graft rejection. Invest Dermatol 2008; 128: 1950-1955.

22 Garrod KR, Liu FC, Forrest LE, Parker I, Kang SM, Cahalan MD. NK cell patrolling and elimination of donor-derived dendritic cells favor indirect alloreactivity. J Immunol 2010; 184: 2329-2336.

23 Ingulli E. Mechanism of cellular rejection in transplantation. Pediatr Nephrol 2010; 25: 61-74.

24 Jung KM, Choi EY. Role for CD40 and CD40L expression in generating CD8 $\mathrm{T}$ cell response to minor histocompatibility antigen, H60. Immune Netw 2007; 7: 173-178.

25 Ryu S, Jung K, Yoo H, Kim T, Kim S, Chang J et al. Cognate CD4 help is essential for the reactivation and expansion of CD8 memory $T$ cells directed against the haematopoietic cell-specific dominant minor histocompatibility antigen, H60. Blood 2009; 113: 4237-4280.

26 Akram A, Inman RD. Immunodominance: a pivotal principle in host response to viral infections. Clin Immunol 2012; 143: 99-115.

27 Chen W, Anton LC, Bennink JR, Yewdell JW. Dissecting the multifactorial causes of immunodominance in class I-restricted $\mathrm{T}$ cell responses to viruses. Immunity 2000; 12: 83-93. 
28 Anderson AC, Nicholson LB, Legge KL, Turchin V, Zaghouani H Kuchroo VK. High frequency of autoreactive myelin proteolipid protein-specific $\mathrm{T}$ cells in the periphery of naive mice: mechanisms of selection of the self-reactive repertoire. J Exp Med 2000; 191: 761-770.

29 Mullbacher A, Lobigs M, Yewdell JW, Bennink JR, Tha Hla R, Blanden RV. High peptide affinity for MHC class I does not correlate with immunodominance. Scand J Immunol 1999; 50: 420-426.

30 Yewdell JW, Bennink JR. Immunodominance in major histocompatibility complex class I-restricted T lymphocyte responses. Annu Rev Immunol 1999; 17: 51-88. (c) (i) (2) (2) This work is licensed under a Creative Commons Attribution-NonCommercial-ShareAlike

3.0

Unported License. The images or other third party material in this article are included in the article's Creative Commons license, unless indicated otherwise in the credit line; if the material is not included under the Creative Commons license, users will need to obtain permission from the license holder to reproduce the material. To view a copy of this license, visit http://creativecommons.org/licenses/by-nc-sa/3.0/ 